\title{
Frequency-Domain Receivers for Rate-1 Space-Time Block Codes
}

\author{
Mário Marques da Silva ${ }^{1,2,3}$, Rui Dinis ${ }^{1,4}$, Américo M. C. Correia ${ }^{1,5}$ \\ ${ }^{1}$ Instituto de Telecomunicações, Lisbon, Portugal \\ ${ }^{2}$ Centro de Estudos de Sistemas de Informação e Tecnologias Informáticas, Portugal \\ ${ }^{3}$ Universidade Autónoma de Lisboa, Lisboa, Portugal \\ ${ }^{4}$ Universidade Nova, Lisboa, Portugal \\ ${ }^{5}$ Instituto Superior de Ciências do Trabalho e da Empresa, Instituto Universidade de Lisboa, Lisboa, Portugal \\ E-mail:marques.silva@ieee.org,rdinis@netcabo.pt,americo.correia@iscte.pt \\ Received September 24, 2009; revised October 28, 2009; accepted November 30, 2009
}

\begin{abstract}
This paper considers iterative frequency-domain receivers for block transmission techniques with rate-1 Space Time Block Coding (STBC) for two and four transmit antennas using both Orthogonal Frequency Division Multiplexing (OFDM) and Single-Carrier (SC) schemes. The proposed receiver includes an interference canceller which enhances the performance of the non-orthogonal STBC scheme with 4 transmit antennas, allowing performances close to those of orthogonal codes. Our performance results show that combining STBC with block transmission techniques allows excellent performances.
\end{abstract}

Keywords: SC-FDE, Turbo Equalization, STBC, OFDM

\section{Introduction}

Block transmission techniques, with appropriate cyclic prefixes and employing FDE techniques (FrequencyDomain Equalization), have been shown to be suitable for high data rate transmission over severely time-dispersive channels [1,2]. OFDM (Orthogonal Frequency Division Multiplexing) is the most popular modulation based on this technique.

Single Carrier modulation using FDE is an alternative approach based on this principle. As with OFDM, the data blocks are preceded by a cyclic prefix, long enough to cope with the overall channel length. Due to the lower envelope fluctuations of the transmitted signals, and implicitly a lower PMEPR (Peak-to-Mean Envelope Power Ratio), Single Carrier - Frequency Domain Equalization (SC-FDE) schemes (also named as Single Carrier-Frequency Domain Multiple Access (SC-FDMA) are especially interesting for the uplink transmission (i.e., the transmission from the mobile terminal to the base station) $[1,2]$.

OFDM transmission technique has been selected for the downlink of Long Term Evolution (LTE) in Release 8 of Third Generation Partnership Project (3GPP), as opposed to WCDMA which is the air interface technique that has been selected by European Telecommunications Standard Institute (ETSI) for UMTS. Moreover, SC-FDE technique has been selected for the uplink of LTE in Release 8 of 3GPP, to be deployed in 2010 .

A promising Iterative Block-Decision Feedback Equalization technique (IB-DFE) for SC-FDE was proposed in [3] and extended to other scenarios in [4] and [5]. These IB-DFE receivers can be regarded as iterative DFE receivers where the feedforward and the feedback operations are implemented in the frequency domain, enhancing the performance as compared to non-iterative methods [3-5].

Transmit Diversity (TD) techniques are particularly interesting for fading channels where it is difficult to have multiple receive antennas (as in conventional receiver diversity schemes). A possible scenario is the downlink transmission where the base station uses several transmittal antennas and the mobile terminal has a single one [6,7].

The application of Alamouti like transmit diversity in OFDM schemes is more-or-less straightforward [8]. With respect to SC-FDE schemes, [9] proposed a way of combining it with a linear FDE. This technique was extended to SC-FDE with IB-DFE in [10].

In this paper, we consider transmit diversity schemes for both OFDM and SC-FDE schemes, specifically the STBC with two [6,7] and four antennas [11,12]. The same concept can be used in STBC based Multiple Input Multiple Output (MIMO) schemes by adopting receive 
diversity. For OFDM schemes we consider conventional receiver and for SC-FDE schemes we consider IB-DFE receivers. For non-orthogonal codes (i.e., with more than two transmit antennas), we also consider iterative receivers with cancellation of the residual interference (for $\mathrm{SC}$ schemes with IB-DFE receivers, this means a negligible increase on the receiver complexity).

This paper is organized as follows. The system considered in this paper is introduced in Section 2 and Section 3 describes the proposed iterative receiver structure for SC-FDE systems with transmit diversity. A set of performance results is presented in Section 4 and Section 5 contains the conclusions of this paper.

\section{System Characterization}

\subsection{Space Time Block Coding for Two Antennas}

We consider block transmission schemes and the lth transmitted block has the form

$$
s_{l}(t)=\sum_{n=-N_{G}}^{N-1} s_{n, l} h_{T}\left(t-n T_{S}\right)
$$

with $T_{s}$ denoting the symbol duration, $N_{G}$ denoting the number of samples at the cyclic prefix and $h_{T}(t)$ is the adopted pulse shaping filter. For a single transmit antenna system, the signal $S_{l}(t)$ is transmitted over a time-dispersive channel and the signal at the receiver input is sampled and the cyclic prefix is removed, leading to the time-domain block $\left\{y_{n, l} ; n=0,1, \ldots, N-1\right\}$, which is then subject to the frequency domain equalization. For SC-FDE schemes the lth time-domain block to be transmitted is $\left\{s_{n, l} ; n=0,1, \ldots, N-1\right\}$, where $S_{n, l}$ is the $n$th data symbol, selected from a given constellation (e.g., a QPSK constellation) under an appropriate mapping rule (it is assumed that $s_{-n, l}=s_{N-n, l}, \quad n=-N_{G},-N_{G}+1, \ldots,-1$ ); the frequency-domain blocks associated with the data are $\left\{S_{k, l} ; k=0,1, \ldots, N-1\right\}=D F T\left\{s_{n, l} ; n=0,1, \ldots, N-1\right\}$. For OFDM schemes, the data symbols are transmitted in the frequency domain, i.e., $S_{k, l}$ are selected according to an appropriate constellation. At the output of the FDE we have the samples $\tilde{A}_{k, l}=Y_{k, l} H_{k, l}^{*} /\left(\alpha+\left|H_{k, l}\right|^{2}\right)$. In the OFDM case this equalization process is simply accomplished through $\tilde{A}_{k, l}=Y_{k, l} H_{k, l}^{*}$.

If we employ Alamouti's transmit diversity we need some processing at the transmitter. The Alamouti's coding can be implemented either in the time domain or in the frequency domain. In this paper we consider timedomain coding, although the extension to frequency domain coding is straightforward. By considering the Space Time Block Coding with two transmit antennas, the time-domain blocks to be transmitted by the $m$ th antenna $(m=1$ or 2$)$ are $\left\{s_{n, l}^{(m)} ; n=0,1, \ldots, N-1\right\}$, with

$$
\begin{aligned}
& s_{n, 2 l-1}^{(1)}=a_{n, 2 l-1} \\
& s_{n, 2 l-1}^{(2)}=-a_{n, 2 l}^{*} \\
& s_{n, 2 l}^{(1)}=a_{n, 2 l} \\
& s_{n, 2 l}^{(2)}=a_{n, 2 l-1}^{*}
\end{aligned}
$$

Considering the matrix-vector representation, this is equivalent to

$$
\mathbf{A}_{n,[1,2]}=\left[\begin{array}{cc}
a_{n, 1} & a_{n, 2} \\
-a_{n, 2}^{*} & a_{n, 1}^{*}
\end{array}\right]
$$

Assuming that the cyclic prefix is longer than the overall channel impulse response of each channel, the $l$ th frequency-domain block after the FDE block (i.e., the DFT of the lth received time-domain block, after removing the cyclic prefix) is

$$
\left\{y_{n, l} ; n=0,1, \ldots, N-1\right\}=\operatorname{IDFT}\left\{Y_{k, l} ; k=0,1, \ldots, N-1\right\},
$$

with

$$
Y_{k, l}=S_{k, l}^{(1)} H_{k, l}^{(1)}+S_{k, l}^{(2)} H_{k, l}^{(2)}+N_{k, l}
$$

where $\left\{H_{k, l}^{(m)} ; k=0,1, \ldots, N-1\right\}=D F T\left\{h_{n, l}^{(m)} ; n=0,1, \ldots, N-1\right\}$ denotes the channel frequency response for the $k$ th subcarrier and the $m$ th transmit antenna (the channel is assumed invariant in the frame) and $N_{k, l}$ is the frequency-domain block channel noise for that subcarrier and the lth block. Assuming, for now, the conventional linear FDE for SC schemes, the Alamouti's post-processing for two antennas (denoted in this paper STBC2) comes,

$$
\begin{aligned}
& \tilde{A}_{k, 2 l-1}=\left[Y_{k, 2 l-1} H_{k, l}^{(1) *}+Y_{k, 2 l}^{*} H_{k, l}^{(2)}\right] \beta_{k}^{(2)} \\
& \tilde{A}_{k, 2 l}=\left[Y_{k, 2 l} H_{k, l}^{(1)^{*}}-Y_{k, 2 l-1}^{*} H_{k, l}^{(2)}\right] \beta_{k}^{(2)}
\end{aligned}
$$

where $\left\{A_{k, m}, k=0,1 \ldots, N\right\}=D F T\left\{a_{n, m}, n=0,1 \ldots, N\right\}$ and where $\beta_{k}^{(2)}=\left(\alpha+\left(\left|H_{k, l}^{(1)}\right|^{2}+\left|H_{k, l}^{(2)}\right|^{2}\right)\right)^{-1}$. This leads to $\tilde{A}_{k, 2 l-j}=A_{k, 2 l-j} \sum_{m=1}^{M}\left|H_{k, l}^{(m)}\right|^{2} \beta_{k}^{(2)}+N_{k, 2 l-j}^{e q} \quad j=0,1$. In addition, we define $\alpha=E\left[\left|N_{k, l}\right|^{2}\right] / E\left[\left|S_{k, 2 l-j}\right|^{2}\right] . \quad N_{k, l}^{e q}$ denotes the equivalent noise for detection purposes, with $E\left[\left|N_{k, l}^{e q}\right|^{2}\right]=\left[2 \sigma_{N}^{2}\left(\sum_{m=1}^{M}\left|H_{k, l}^{(m)}\right|^{2}\right)\right] \beta_{k}^{(2)}$, and with $\sigma_{N}^{2}=E\left[\left|N_{k, l}\right|^{2}\right] / 2$.

The Alamouti's post-processing for OFDM signals is the same as defined in (5) but without multiplying by the $\beta_{k}^{(2)}$ component. 


\subsection{Space Time Block Coding for Four Antennas}

Using unspecified complex valued modulation, such an improvement is possible only for the two antenna scheme. Higher schemes with 4 and 8 antennas with code rate one exists only in the case of binary transmission [13]. The proposed STBC4 scheme has $M=4$ transmit antennas, presenting a code rate one. The symbol construction can be generally written as [11-12]

$$
\mathbf{A}_{n,[1,4]}=\left[\begin{array}{cc}
\mathbf{A}_{n,[1,2]} & \mathbf{A}_{n,[3,4]}^{*} \\
\mathbf{A}_{n,[3,4]} & -\mathbf{A}_{n,[1,2]}^{*}
\end{array}\right]
$$

where $\mathbf{A}_{n,[3,4]}$ is the same as $\mathbf{A}_{n,[1,2]}$, by replacing the subscripts 1 by 3 and 2 by 4 . Similarly to (2), considering the Space Time Block Coding with four transmit antennas, the time-domain blocks to be transmitted by the $m$ th antenna $(m=1,2,3$ or 4$)$ are $\left\{s_{n, l}^{(m)} ; n=0,1, \ldots, N-1\right\}$, with

$$
\begin{array}{llll}
s_{n, 4 l-3}^{(1)}=a_{n, 4 l-3} & s_{n, 4 l-2}^{(1)}=a_{n, 4 l-2} & s_{n, 4 l-1}^{(1)}=a_{n, 4 l-1} & s_{n, 4 l}^{(1)}=a_{n, 4 l} \\
s_{n, 4 l-3}^{(2)}=-a_{n, 4 l-2}^{*} & s_{n, 4 l-2}^{(2)}=a_{n, 4 l-3}^{*} & s_{n, 4 l-1}^{(2)}=-a_{n, 4 l}^{*} & s_{n, 4 l}^{(2)}=a_{n, 4 l-1}^{*} \\
s_{n, 4 l-3}^{(3)}=a_{n, 4 l-1}^{*} & s_{n, 4 l-2}^{(3)}=a_{n, 4 l}^{*} & s_{n, 4 l-1}^{(3)}=-a_{n, 4 l-3}^{*} & s_{n, 4 l}^{(3)}=-a_{n, 4 l-2}^{*} \\
s_{n, 4 l-3}^{(4)}=-a_{n, 4 l} & s_{n, 4 l-2}^{(4)}=a_{n, 4 l-1} & s_{n, 4 l-1}^{(4)}=a_{n, 4 l} & s_{n, 4 l}^{(4)}=-a_{n, 4 l-3}
\end{array}
$$

The lth frequency-domain block after the FDE block (i.e., the DFT of the $l$ th received time-domain block, after removing the cyclic prefix) is $\left\{y_{n, l} ; n=0,1, \ldots, N-1\right\}=$ $\operatorname{IDFT}\left\{Y_{k, l} ; k=0,1, \ldots, N-1\right\}$, with

$$
Y_{k, l}=S_{k, l}^{(1)} H_{k, l}^{(1)}+S_{k, l}^{(2)} H_{k, l}^{(2)}+S_{k, l}^{(3)} H_{k, l}^{(3)}+S_{k, l}^{(4)} H_{k, l}^{(4)}+N_{k, l}
$$

Assuming, for now, the conventional SC-FDE decoding (i.e., no IB-DFE receiver), the post-processing STBC for four antennas $(M=4)$ comes,

$$
\begin{aligned}
& \tilde{A}_{k, 4 l-3}=\left[Y_{k, 4 l-3} H_{k, l}^{(1)^{*}}+Y_{k, 4 l-2}^{*} H_{k, l}^{(2)}-Y_{k, 4 l-1}^{*} H_{k, l}^{(3)}-Y_{k, 4 l} H_{k, l}^{(4) *}\right] \beta_{k}^{(4)}= \\
& =\overbrace{A_{k, 4 l-3} \sum_{m=1}^{M}\left|H_{k, l}^{(m)}\right|^{2}}^{\text {Desired Symbol }} \overbrace{C_{k} A_{k, 4 l}}^{\text {Re sidual Interference }}+N_{k, 4 l-3}^{e q} \\
& \tilde{A}_{k, 4 l-2}=\left[Y_{k, 4 l-2} H_{k, l}^{(1)^{*}}-Y_{k, 4 l-3}^{*} H_{k, l}^{(2)}-Y_{k, 4 l}^{*} H_{k, l}^{(3)}+Y_{k, 4 l-1} H_{k, l}^{(4) *}\right] \beta_{k}^{(4)}= \\
& =A_{k, 4 l-2} \sum_{m=1}^{M}\left|H_{k, l}^{(m)}\right|^{2}+C_{k} A_{k, 4 l-1}+N_{k, 4 l-2}^{e q} \\
& \tilde{A}_{k, 4 l-1}=\left[Y_{k, 4 l-1} H_{k, l}^{(1)^{*}}+Y_{k, 4 l}^{*} H_{k, l}^{(2)}+Y_{k, 4 l-3}^{*} H_{k, l}^{(3)}+Y_{k, 4 l-2} H_{k, l}^{(4) *}\right] \beta_{k}^{(4)}= \\
& =A_{k, 4 l-1} \sum_{m=1}^{M}\left|H_{k, l}^{(m)}\right|^{2}+C_{k} A_{k, 4 l-2}+N_{k, 4 l-1}^{e q} \\
& \tilde{A}_{k, 4 l}=\left[Y_{k, 4 l} H_{k, l}^{(1) *}-Y_{k, 4 l-1}^{*} H_{k, l}^{(2)}+Y_{k, 4 l-2}^{*} H_{k, l}^{(3)}-Y_{k, 4 l-3} H_{k, l}^{(4) *}\right] \beta_{k}^{(4)}= \\
& =A_{k, 4 l} \sum_{m=1}^{M}\left|H_{k, l}^{(m)}\right|^{2}-C_{k} A_{k, 4 l-3}+N_{k, 4 l}^{e q}
\end{aligned}
$$

with $\beta_{k}^{(4)}=\left(\alpha+\sum_{m=1}^{M}\left|H_{k, l}^{(m)}\right|^{2}\right)^{-1}$, where is defined as above $(j=1,2,3,4)$, and where $C_{k}=2 \operatorname{Re}\left\{H_{k, l}^{(1) *} H_{k, l}^{(4)}-H_{k, l}^{(2)} H_{k, l}^{(3) *}\right\} /$ $\left\{\left(\sum_{m=1}^{M}\left|H_{k, l}^{(m)}\right|^{2}\right)\right\}$ which stands for the residual interference coefficient generated in the STBC decoding process. In the following we will show how we can remove this residual interference.

\section{Receiver Design}

In this section we describe an IB-DFE receiver for Space Time Block Coding with four antennas considering SC-FDE signals. The frequency-domain block at the output of the receiver is $\left\{\tilde{A}_{k, 4 l-j}^{(i)} ; k=0,1, \ldots, N-1\right\}$, with

$$
\begin{aligned}
& \tilde{A}_{k, 4 l-3}^{(i)}=\overbrace{Y_{k, 4 l-3} F_{k, l}^{(i)(1)}+Y_{k, 4 l-2}^{*} F_{k, l}^{(i)(2)}-Y_{k, 4 l-1}^{*} F_{k, l}^{(i)(3)}-Y_{k, 4 l} F_{k, l}^{(i)(4)}}^{\text {STBC4 decoding plus IB-DFE feedforward }} \\
& \text { Cancellation of residual interference IB-DFE feedback } \\
& +\quad \overbrace{+C_{k} \bar{A}_{k, 4 l}^{(i)}}-\overbrace{B_{k, l}^{(i)} \bar{A}_{k, 4 l-3}^{(i-1)}}^{v_{n}} \\
& \tilde{A}_{k, 4 l-2}^{(i)}=Y_{k, 4 l-2} F_{k, l}^{(i)(1)}-Y_{k, 4 l-3}^{*} F_{k, l}^{(i)(2)}-Y_{k, 4 l}^{*} F_{k, l}^{(i)(3)}+Y_{k, 4 l-1} F_{k, l}^{(i)(4)} \\
& -C_{k} \widehat{A}_{k, 4 l-1}^{(i)}-B_{k, l}^{(i)} \bar{A}_{k, 4 l-2}^{(i-1)} \\
& \tilde{A}_{k, 4 l-1}^{(i)}=Y_{k, 4 l-1} F_{k, l}^{(i)(1)}+Y_{k, 4 l}^{*} F_{k, l}^{(i)(2)}+Y_{k, 4 l-3}^{*} F_{k, l}^{(i)(3)}+Y_{k, 4 l-2} F_{k, l}^{(i)(4)} \\
& -C_{k} \widehat{A}_{k, 4 l-2}^{(i)}-B_{k, l}^{(i)} \bar{A}_{k, 4 l-1}^{(i-1)} \\
& \widehat{A}_{k, 4 l}^{(i)}=Y_{k, 4 l} F_{k, l}^{(i)(1)}-Y_{k, 4 l-1}^{*} F_{k, l}^{(i)(2)}+Y_{k, 4 l-2}^{*} F_{k, l}^{(i)(3)}-Y_{k, 4 l-3} F_{k, l}^{(i)(4)} \\
& +C_{k} \widehat{A}_{k, 4 l-3}^{(i)}-B_{k, l}^{(i)} \bar{A}_{k, 4 l}^{(i-1)}
\end{aligned}
$$

where $C_{K}$ is as defined for (9). The feedforward coefficients are $\left\{F_{k, l}^{(i)(m)} ; k=0,1, \ldots, N-1 ; m=1,2, \ldots, M\right\}$ and the feedback coefficients are $\left\{B_{k, l}^{(i)} ; k=0,1, \ldots, N-1\right\}$. The $\operatorname{block}\left\{\hat{A}_{n, 4 l-j}^{(i-1)} ; n=0,1, \ldots, N-1\right\}=D F T\left\{\widehat{a}_{n, 4 l-j}^{(i-1)} ; n=0,1, \ldots, N-1\right\}$, and denotes the DFT transform of the data estimates associated to the previous iteration, i.e., the Hard Decisions associated to the time-domain block at the output of $\left\{\tilde{a}_{n, 4 l-j}^{(i-1)} ; n=0,1, \ldots, N-1\right\}=\operatorname{IDFT}\left\{\tilde{A}_{k, 4 l-j}^{(i-1)} ; k=0,1, \ldots, N-1\right\}$. $\left\{\bar{A}_{k, 4 l-j}^{(i-1)} ; k=0,1, \ldots, N-1 ; j=0,1,2,3\right\}$ denotes the average signal conditioned to the FDE output for the previous iteration $\left\{\bar{a}_{n, 4 l-j}^{(i-1)} ; n=0,1, \ldots, N-1\right\}$ from (19). It is worth noting that since $\tilde{A}_{k, 4 l-j}^{(i)}$ presents residual interference, the detection of $A_{k, 4 l-j}^{(i)}$ should be accompanied by the detection of $A_{k, 4 l-p}^{(i)}$ (with $p=3-j$ ) to allow the cancellation of the residual interference generated in the STBC4 
decoding process.

In case of a SISO system, (10) takes the form $\widehat{A}_{k, l}^{(i)}=Y_{k, l} F_{k, l}^{(i)}-B_{k, l}^{(i)} \bar{A}_{k, l}^{(i-1)}$, i.e., there is a single branch (there is no STBC4 decoding) and there is no cancellation of the residual interference. In case of STBC2 (two transmit antennas), there is no residual interference component.

To further improve performance with STBC4 the residual interference to be subtracted (which is a function of the estimate of the symbol that generates interference), we consider an Iterative Interference Cancellation (IIC) that can be implemented as follows:

1) Compute $\widehat{A}_{k, 4 l-j}^{(i)(q)}$ using (10) without cancelling the residual interference.

2) Based on $\widehat{A}_{k, 4 l-j}^{(i)(q)}$ from i., compute $\widehat{A}_{k, 4 l-p}^{(i)(q)}$ after cancelling the corresponding residual interference.

3) Based on $\hat{A}_{k, 4 l-p}^{(i)(q)}$ from ii., compute $\widehat{A}_{k, 4 l-j}^{(i)(q+1)}$ after cancelling the residual interference $\left(C_{k} \widehat{A}_{k, 4 l-p}^{(i)}\right)$.

4) Repeat steps ii. and iii. iteratively to improve the accuracy of $\widehat{A}_{k, 4 l-p}^{(i)}$ (cancellation of the residual interference), which will finally be used to improve the accuracy of $\widehat{A}_{k, 4 l-j}^{(i)}$.

It can be shown that the optimum feedback coefficients are described by [3-4].

It can be shown that the optimum feedback coefficients are described by [3-4].

$$
B_{k, l}^{(i)}=\sum_{m=1}^{M} F_{k, l}^{(i)(m)} H_{k, l}^{(m)}-1
$$

and the feedforward coefficients are given by

$$
F_{k, l}^{(i)(m)}=\frac{Q_{k, l}^{(m)}}{\left[\alpha+\left(1-\left(\rho_{l}^{(i-1)}\right)^{2}\right) \sum_{m=1}^{M}\left|H_{k, l}^{(m)}\right|^{2}\right] \gamma_{l}^{(i)}}
$$

with $Q_{k, l}^{(m)}=H_{k, l}^{(m)^{*}}$ for $m=1$ or 4 and $Q_{k, l}^{(m)}=H_{k, l}^{(m)}$ for $m=2$ or 3. In the particular case of SISO we only have $m=1$ (with $M=1$ ) and $Q_{k, l}=H_{k, l}^{*}$. In case of STBC of order two (i.e., STBC2), we have $Q_{k, l}^{(m)}=H_{k, l}^{(m)^{*}}$ for $m=1$ and $Q_{k, l}^{(m)}=H_{k, l}^{(m)}$ for $m=2$. The parameter $\gamma_{l}^{(i)}$ is defined as

$$
\gamma_{l}^{(i)}=\frac{1}{N} \sum_{m=1}^{M} \sum_{k=0}^{N-1} F_{k, l}^{(i)(m)} H_{k, l}^{(m)}
$$

and the correlation factor $\rho_{4 l-j}^{(i-1)}$ is defined as

$$
\rho_{4 l-j}^{(i-1)}=\frac{E\left[\hat{a}_{n, 4 l-j}^{(i-1)} a_{n, 4 l-j}^{*}\right]}{E\left[\left|a_{n, 4 l-j}\right|^{2}\right]}
$$

It can be shown that, for the QPSK modulation, the correlation coefficient is given by [14]

$$
\rho_{4 l-j}^{(i)}=\frac{1}{2 N} \sum_{n=0}^{N-1}\left(\rho_{n, 4 l-j}^{I(i)}+\rho_{n, 4 l-j}^{Q(i)}\right)
$$

( $\rho_{4 l-j}^{(i)}$ is almost independent of $l$ for large values of $N$, provided that $H_{k, l}^{(m)}$ is constant for the frame duration), as

$$
\begin{aligned}
& \rho_{n, 4 l-j}^{I(i)}=\left|\tanh \left(\frac{L_{n}^{I(i)}}{2}\right)\right| \\
& \rho_{n, 4 l-j}^{Q(i)}=\left|\tanh \left(\frac{L_{n}^{Q(i)}}{2}\right)\right|
\end{aligned}
$$

The LLRs (Log Likelihood Ratios) of the "in-phase bit" and the "quadrature bit", associated to $a_{n, 4 l-j}^{I(i)}$ and $a_{n, 4 l-j}^{Q(i)}$, respectively, are given by

$$
\begin{aligned}
& L_{n}^{I(i)}=\frac{2}{\sigma_{i}^{2}} \tilde{a}_{n, 4 l-j}^{I(i)} \\
& L_{n}^{Q(i)}=\frac{2}{\sigma_{i}^{2}} \tilde{a}_{n, 4 l-j}^{Q(i)}
\end{aligned}
$$

respectively, with

$$
\sigma_{i, 4 l-j}^{2}=\frac{1}{2} E\left[\left|a_{n, 4 l-j}-\tilde{a}_{n, 4 l-j}^{(i)}\right|^{2}\right] \approx \frac{1}{2 N} \sum_{n=0}^{N-1}\left|\hat{a}_{n, 4 l-j}^{(i)}-\tilde{a}_{n, 4 l-j}^{(i)}\right|^{2}
$$

(as with $\rho_{4 l-j}^{(i)}, \sigma_{i, 4 l-j}^{2}$ is almost independent of $l$ for large values of $N$, provided that $H_{k, l}^{(m)}$ remains constant for the frame duration).

The conditional average values associated with the data symbols are given by

$$
\bar{a}_{n, 4 l-j}^{(i)}=\tanh \left(\frac{L_{n, 4 l-j}^{I(i)}}{2}\right)+j \tanh \left(\frac{L_{n, 4 l-j}^{Q(i)}}{2}\right)
$$

Therefore, the several symbols of order $j$ th $(j=0,1,2,3)$ that comprise the STBC4 block need to be decoded independently by the IB-DFE receiver, with the exception of the symbol estimates that originate the residual interference generated in the STBC4 decoding process, as shown in (10). The IB-DFE with soft decisions described above does not need to perform the channel decoding in the feedback loop. As an alternative, we can define a Turbo FDE that employs the channel decoder outputs, instead of the uncoded "soft decisions" in the feedback loop of the IB-DFE. The main difference between IB-DFE with soft decisions and the Turbo FDE is in the decision device: in the first case the decision device is a symbol-by-symbol soft-decision (for QPSK constellation this corresponds to the hyperbolic tangent, as in (19)); 
for the Turbo FDE a Soft-In, Soft-Out channel decoder is employed in the feedback loop. The Soft-In, Soft-Out block, that can be implemented as defined in [15], provides the LLRs of both the "information bits" and the "coded bits". The input of the Soft-In, Soft-Out block are LLRs of the "coded bits" at the FDE output, given by (17) and (18).

The receiver for OFDM schemes with STBC2 is straightforward. For OFDM schemes with STBC4, (10) also applies with the difference that there is no feedback component, and the feedforward component only have the numerator of (12). It is worth noting that these STBC schemes can easily be extended to multiple receive antennas.

\section{Performance Results}

In this section we present a set of performance results concerning the proposed receivers, for both SC-FDE and OFDM schemes with two and four-antenna STBC schemes. We consider both Bit Error Rate (BER) and Block Error Rate (BLER) performances, which are expressed as a function of $E_{b} / N_{0}$, where $N_{0}$ is the one-sided power spectral density of the noise and $E_{b}$ is the energy of the transmitted bits (i.e., the degradation due to the useless power spent on the cyclic prefix is not included).

Each block has $N=256$ symbols selected from a QPSK constellation under a Gray mapping rule (similar results were observed for other values of $N$, provided that $N>>1$ ). The pulse shaping filter is raised cosine with roll-off 0.1 . The results shown in this paper considers the Pedestrian A propagation environment [16].

The channel is assumed to be invariant during the block. The duration of the useful part of the blocks $(N$ symbols) is $1 \mu \mathrm{s}$ and the cyclic prefix has duration $0.125 \mu \mathrm{s}$. For SC-FDE systems we considered the IBDFE receiver with soft decisions and the Turbo FDE, both with five iterations. Beyond this number the performance improvement was almost negligible.

Linear power amplification is considered at the transmitter and perfect synchronization is assumed at the receiver. The channel encoder is a convolutional code with generators $1+D^{2}+D^{3}+D^{5}+D^{6}$ and $1+D+D^{2}+D^{3}+D^{6}$, and the coded bits associated to a given block are interleaved and mapped into the constellation points.

Figure 1 considers uncoded BER results for the SCFDE and a linear FDE receiver (i.e., just the first iteration of the IB-DFE receiver) versus the IB-DFE receiver with soft decisions (i.e., without channel decoding in the feedback loop), in this case with five iterations. Clearly, the increased diversity due to STBC schemes leads to significant performance improvements relatively to the SISO case. From this figure, it is also clear that the IB-DFE performs always better than the linear FDE receiver. It can also be observed that the STBC4 with the linear FDE receiver performs very badly, due to the residual interference (generated in the STBC4 decoding process). However, when we add the IB-DFE with soft decisions to the STBC4, we have a significant performance improvement, namely due to the ability to mitigate the residual interference. It is worth noting that, with the IB-DFE receiver, the STBC4 achieves a performance improvement over the STBC2. It happens because the proposed receiver cancels the interference generated in the STBC4 decoding process. This residual interference is, in fact, the reason why this STBC4 scheme is considered as non-orthogonal. In this case, we have seen that the non-orthogonality is not a reason for loss of performance.

Figure 2 concerns the coded results for the SC-FDE. In this case, the Linear FDE and the Turbo FDE receiveris considered. For the linear FDE receiver, the STBC4 performs worse than the STBC2, due to the residual interference. However, for the Turbo FDE (i.e., the proposed iterative frequency-domain receiver that employs the channel decoder outputs), the STBC4 outperforms

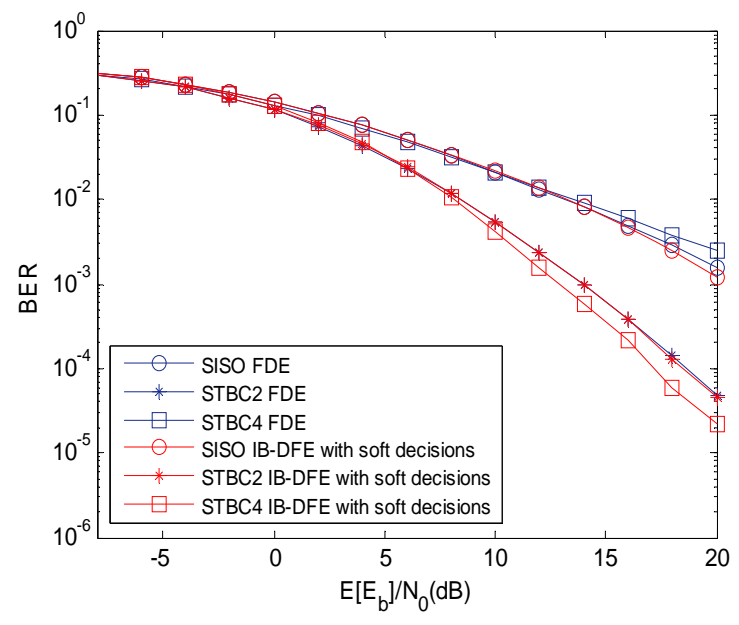

Figure 1. Uncoded BER results for the SC-FDE.

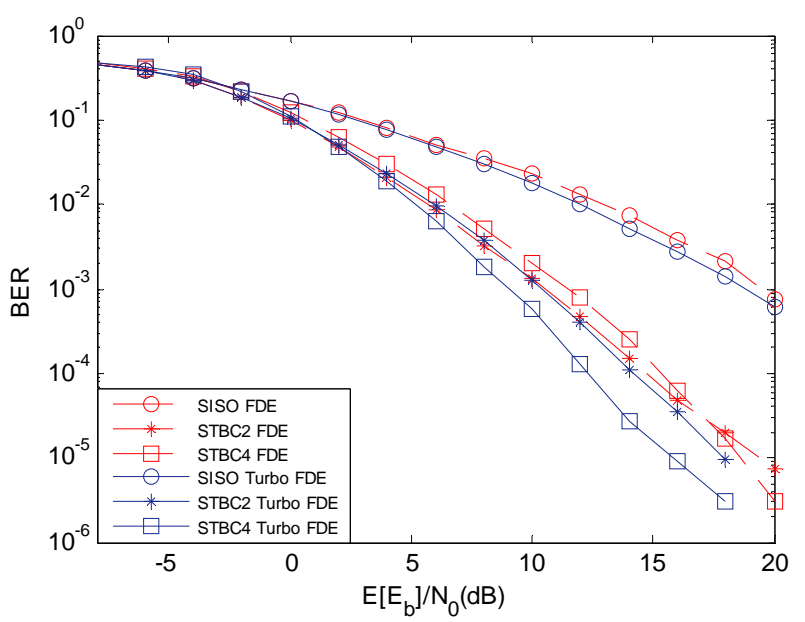

Figure 2. Coded BER results for the SC-FDE. 


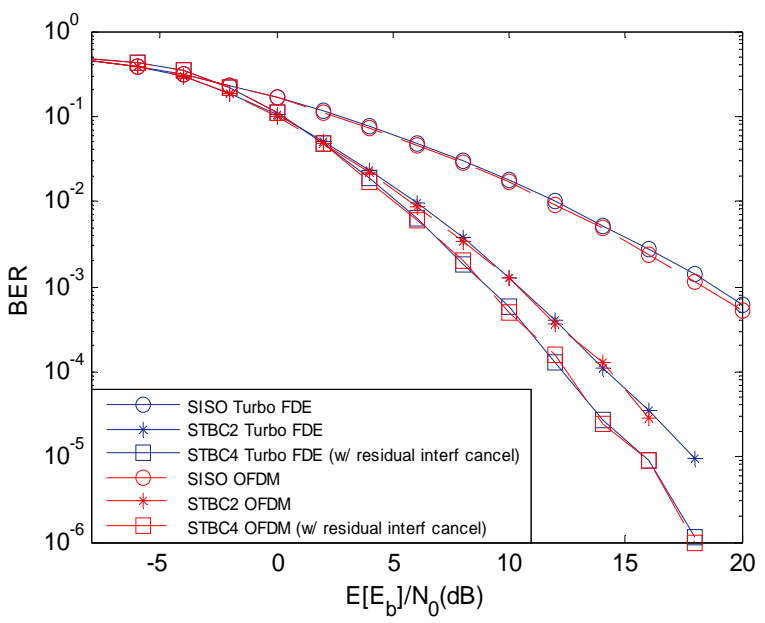

Figure 3. Coded BER for SC-FDE and OFDM.

the STBC2 (and the SISO, as expected). This is a consequence of the additional diversity order and the effective residual interference cancellation inherent to the proposed receiver. Therefore, although using a higher number of antennas leads to an increase in the system complexity, its advantage is clear as long as the proposed iterative receiver is adopted.

Figure 3 shows a performance comparison between SC-FDE and OFDM when channel coding is considered (it is well-know that uncoded performances are very poor for OFDM schemes). Note that the OFDM receiver for the STBC4 also includes a residual interference canceller, similar to the one included and described in the IB-DFE that was considered for the SC-FDE STBC4. The proposed Turbo FDE receiver for SC-FDE signals allows similar or better performance than coded OFDM signals for the STBC schemes considered. However, OFDM technique presents much more demanding requirements in terms of PMEPR, as compared to SC-FDE technique.

Figure 4 shows the uncoded BER performance of STBC4 with and without residual interference cancellation for both SC-FDE (in this case the IB-DFE receiver is considered) and OFDM. From this figure it is seen that, when the residual interference cancellation is considered, SC-FDE with the proposed iterative receiver achieves better results than those achieved with OFDM. Moreover, when we focus on the results without the residual interference cancellation, it is clear the much better results achieved with the SC-FDE due to the inherent ability of the iterative frequency domain SC-FDE receiver to cancel generic interference. In this case, SC-FDE without the residual interference cancellation achieves approximately the same performance than that achieved with the OFDM scheme with the interference cancelled. Finally, it is noticeable the very bad performance obtained with the OFDM technique when the residual interference is not cancelled.

Figure 5 shows the coded BER performance of STBC4 with and without residual interference cancella- tion for both SC-FDE and OFDM. From this figure it is observed that, when the residual interference cancellation is considered, SC-FDE with the proposed iterative receiver (i.e., the Turbo FDE receiver) achieves similar results to those achieved with OFDM. However, when we focus on the results without the residual interference cancellation, as before, it is clear the better results achieved with the SC-FDE, for higher values of $E_{b} / N_{0}$, due to the inherent ability of the iterative frequency domain receiver (Turbo FDE) to cancel generic interference. Figure 6 presents results similar to Figure 3 , but in terms of BLER, instead of the BER. As before, for the same diversity order, SC-FDE schemes achieve similar results as those obtained with the OFDM. The BLER results confirm the advantage of the STBC4 over lower diversity orders.

\section{Conclusions}

In this paper we considered iterative frequency-do main

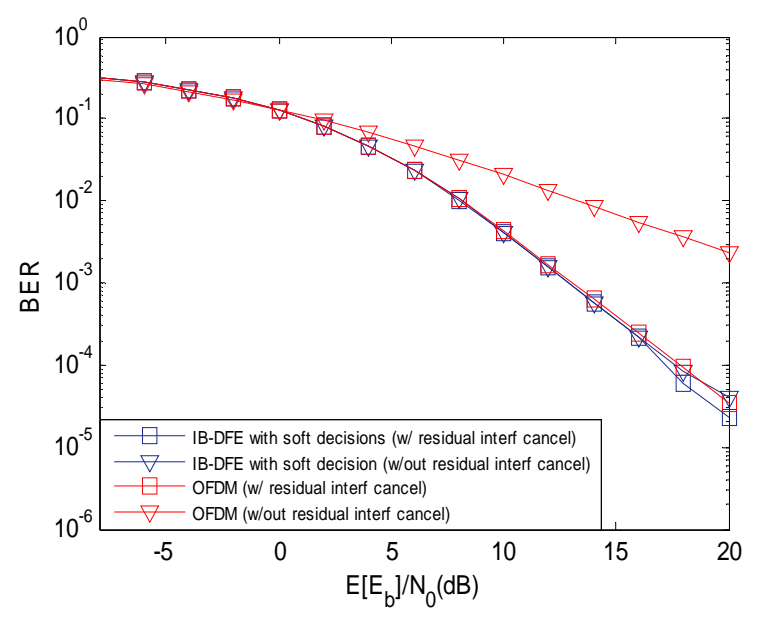

Figure 4. Uncoded BER performance for STBC4 (w/ and w/out residual interference cancellation).

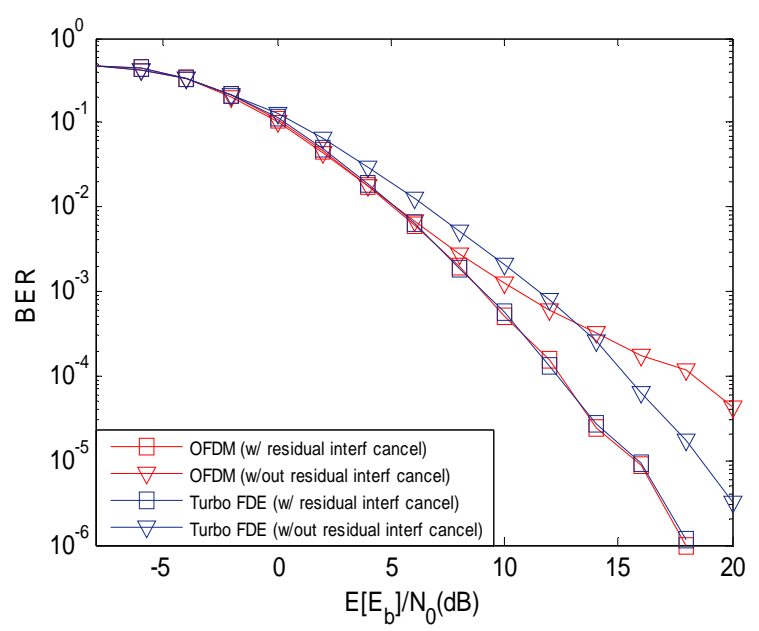

Figure 5. Coded BER performance for STBC4 (w/ and w/out residual interference cancellation). 


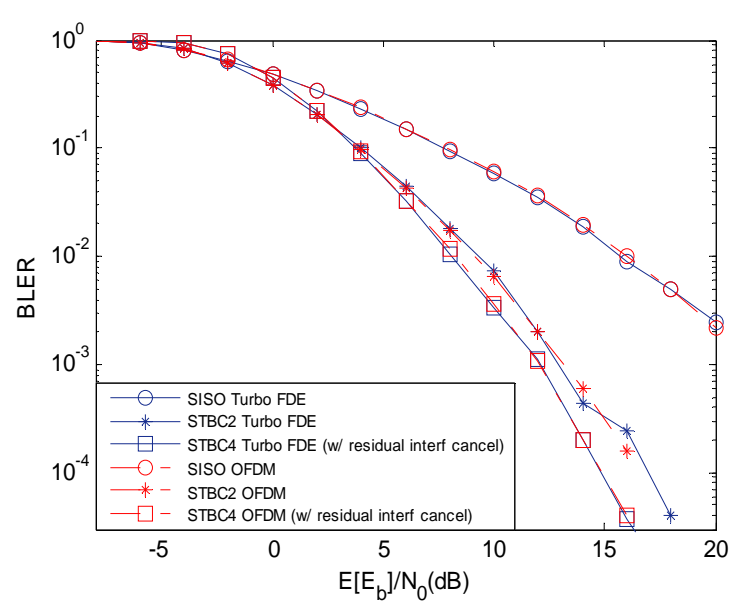

Figure 6. Coded BLER for SC-FDE and OFDM

receivers for SC-FDE technique with code rate-1 STBC using two or four transmit antennas. OFDM technique was also considered in system description and performance results.

Since our STBC with 4 transmit antennas is not orthogonal, our receiver includes the cancellation of the residual interference.

The proposed Turbo FDE receiver for SC-FDE signals allows similar or better performance than coded OFDM signals with the same diversity order. However, OFDM technique presents much more demanding requirements in terms of PMEPR, as compared to SC-FDE technique, limiting its applicability. In this sense, SC-FDE is a good alternative to OFDM transmission technique, especially for the uplink.

It was shown that the best overall performance is achieved with STBC4 schemes, as long as the receiver includes the described residual interference cancellation system. It is worth noting that by adding $N$ order receive diversity ( $N$ receive antennas instead of a single one), the proposed SC-FDE STBC4 receiver keeps being valid and the system can be seen as a $4 \times N$ MIMO system.

\section{Acknowledgements}

This work was supported by the Portuguese Foundation for the Science and Technology (FCT).

\section{References}

[1] A. Gusmão, R. Dinis, J. Conceicão, and N. Esteves, "Comparison of two modulation choices for broadband wireless communications," Proceedings IEEE VTC Spring, pp. 1300-1305, May 2000.

[2] D. Falconer, S. Ariyavisitakul, A. Benyamin-Seeyar, and
B. Eidson, "Frequency domain equalization for singlecarrier broadband wireless systems," IEEE Communications Magazines, Vol. 4, No. 4, pp. 58-66, April 2002.

[3] N. Benvenuto and S. Tomasin, "Block iterative DFE for single carrier modulation," IEE Electronic Letters, Vol. 39, No. 19, September 2002.

[4] R. Dinis, A. Gusmão, and N. Esteves, "On broadband block transmission over strongly frequency-selective fading channels," Wireless 2003, Calgary, Canada, July 2003.

[5] R. Dinis, R. Kalbasi, D. Falconer, and A. Banihashemi, "Iterative layered space-time receivers for single-carrier transmission over severe time-dispersive channels," IEEE Communication Letters, Vol. 8, No. 9, pp. 579-581, September 2004.

[6] S. M. Alamouti, "A simple transmitter diversity scheme for wireless communications," IEEE JSAC, pp. 14511458, October 1998.

[7] Tarokh, et al., "Space-time block codes from orthogonal designs," IEEE Transactions on Information Theory, pp. 1456-1467, July 1999.

[8] J. Wang, O. Wen, S. Li, R. Shu, and K. Cheng, "Capacity of alamouti coded OFDM systems in time-varying multipath rayleigh fading channels," IEEE VTC'06 (Spring), May 2006.

[9] N. Al-Dhahir, "Single-carrier frequency-domain equalization for space-time block-coded transmission over frequency-selective fading channels," IEEE Communications Letters, Vol. 5, July 2001.

[10] R. Dinis, A. Gusmão, and N. Esteves, "Iterative blockDFE techniques for single-carrier-based broadband communications with transmit/receive space diversity," IEEE ISWCS'04, Port Louis, Mauritius, September 2004.

[11] M. M. Silva and A. Correia, "Space time block coding for 4 antennas with coding rate 1," IEEE International Symposium on Spread Spectrum Techniques and Application (ISSSTA), Prague, Check Republic, 2-5 September 2002.

[12] M. M. Silva, A. Correia, and R. Dinis, "Wireless systems on transmission techniques for multi-antenna W-CDMA systems", European Transactions on Telecommunications, Wiley Interscience, DOI: 10.1002/ett.1252, http://dx.doi. org/10.1002/ett.1252, published on-line in advance of print in 16 November 2007.

[13] B. Hochwald, T. Marzetta, and C. Papadias, "A transmitter diversity scheme for wideband CDMA systems based on space-time spreading," IEEE Journal on Selected Area in Communications, Vol. 19, No. 1, pp. 48-60, January 2001.

[14] A. Gusmão, P. Torres, R. Dinis, and N. Esteves, “A class of iterative FDE techniques for reduced-CP SC-based block transmission," International Symposium on Turbo Codes, April 2006.

[15] B. Vucetic and J. Yuan, "Turbo codes: principles and applications," Kluwer Academic Publication, 2002.

[16] ETSI, "Channel models for hiperLAN/2 in different indoor scenarios,” ETSI EP BRAN 3ERI085B; pp. 1-8, 1998. 